\title{
Theory for the ultrafast ablation of graphite films
}

\author{
H. O. Jeschke, M. E. Garcia and K. H. Bennemann \\ Institut für Theoretische Physik der Freien Universität Berlin, Arnimallee 14, 14195 Berlin, Germany,
}

(November 19, 2018)

\begin{abstract}
The physical mechanisms for damage formation in graphite films induced by femtosecond laser pulses are analyzed using a microscopic electronic theory. We describe the nonequilibrium dynamics of electrons and lattice by performing molecular dynamics simulations on time-dependent potential energy surfaces. We show that graphite has the unique property of exhibiting two distinct laser induced structural instabilities. For high absorbed energies $(>3.3 \mathrm{eV} / \mathrm{atom})$ we find nonequilibrium melting followed by fast evaporation. For low intensities above the damage threshold $(>2.0 \mathrm{eV} /$ atom $)$ ablation occurs via removal of intact graphite sheets.

52.38.Mf, 64.70.Hz, 68.35.Ja, 81.05.Uw
\end{abstract}

Material irradiation with femtosecond laser pulses gives rise to many interesting phenomena involving atomic motion, like ultrafast phase transitions [1] [7, ablation [8.9], optical breakdown [10 12], and excitation of coherent phonons 13]. Ultrafast laser ablation, in particular, has been extensively studied mainly because of its important technological applications [14]. However, little is known about the microscopic mechanisms leading to ablation.

We define the ablation threshold as the laser fluence for which lattice instabilities of such magnitude are induced that the system is irreversibly damaged and at least a monolayer of material is removed [10]. On the basis of many experimental studies it is commonly assumed that the ablation process near the threshold is always initiated by the melting (or ultrafast melting) of the material.

In this letter we show that there is an exception to this general rule. We present a theoretical study of the microscopic processes leading to the femtosecond ablation of graphite films. We conclude that, due to its layered, $s p^{2}$-bonded structure, graphite presents the unique property of exhibiting two different ablation mechanisms and therefore two different ablation thresholds. The mechanism for the low fluence ablation threshold is the removal of intact graphite sheets and does not involve melting. In contrast, the high fluence threshold corresponds to bond breaking processes inside the graphite layers and leads to ultrafast melting and expansion of the film. Moreover, we find that the metastable and short-lived low density liquid state formed at the second ablation threshold presents some signatures corresponding to low density liquid carbon (LDLC) 15.

We study the laser-induced ultrafast dynamics of a graphite film by performing molecular dynamics calculations based upon a tight-binding Hamiltonian. The effects of the strong electronic nonequilibrium created by ultrashort laser pulses are accounted for with a method of determining time-dependent electronic occupation numbers, which leads to potential energy surfaces changing with time [3]. The film geometry is simulated with a
MD supercell that has periodic boundary conditions in the horizontal directions, while in the direction perpendicular to this, the material borders on the vacuum [16]. The shape of the MD supercell is constant in this model, and the size is sufficiently large to allow any expansion of the material along the vertical axis. With this method we treat the inertial confinement which is expected to be important in the initial stages of the response of a graphite film to laser excitation. The method of using a column-shaped MD supercell of fixed shape and size should be a good approximation to the physical reality in the first 10 to $100 \mathrm{ps}$ after laser excitation, i.e. as long as the vertical expansion of the material dominates and the horizontal expansion can still be neglected.

In the MD simulations the forces acting on atom $k$ is calculated as $\mathbf{f}_{k}\left(\left\{r_{i j}(t)\right\}, t\right)=-\nabla_{k} \Phi\left(\left\{r_{i j}(t)\right\}, t\right)$, where $r_{i j}=\left|\mathbf{r}_{i}-\mathbf{r}_{j}\right|$ is the distance between atoms $i$ and $j$, and $\Phi\left(\left\{r_{i j}(t)\right\}, t\right)$ is the potential. It is clear that, for the description of laser induced nonequilibrium processes, $\Phi\left(\left\{r_{i j}(t)\right\}, t\right)$ cannot be a model potential, but it has to be derived from a microscopic electronic theory, and is given by

$$
\Phi\left(\left\{r_{i j}(t)\right\}, t\right)=-\sum_{m} n\left(\epsilon_{m}, t\right) \epsilon_{m}-\frac{1}{2} \sum_{\substack{i j \\ j \neq i}} E_{\text {rep }}\left(r_{i j}\right) .
$$

In Eq. (11) the quantities $\epsilon_{m}=\left\langle m\left|H_{\mathrm{TB}}\left(\left\{r_{i j}(t)\right\}\right)\right| m\right\rangle$ are the eigenvalues of the electronic Hamiltonian, $n\left(\epsilon_{m}, t\right)$ the corresponding electronic occupations, and $E_{\mathrm{rep}}\left(r_{i j}\right)$ the repulsive core-core potential. We use a tight-binding Hamiltonian

$$
H_{\mathrm{TB}}=\sum_{i \eta} \epsilon_{i \eta} n_{i \eta}+\sum_{\substack{i j \eta \vartheta \\ j \neq i}} t_{i j}^{\eta \vartheta} c_{i \eta}^{+} c_{j \vartheta} .
$$

Here, $n_{\text {iq }}$ represents the occupation number operator for the orbital $\eta$ of atom $i$, and the hopping matrix elements have been abridged by $t_{i j}^{\eta \vartheta}$. We follow Slater and Koster 17] in their treatment of the direction dependence of the hopping integrals $t_{i j}^{\eta \vartheta}$. We consider the four valence orbitals of carbon $2 s, 2 p_{x}, 2 p_{y}$ and $2 p_{z}$ (thus $\eta, \zeta=s s \sigma$, 
$s p \sigma, p p \sigma$ and $p p \pi)$. For the distance dependence of the TB parameters $V_{s s \sigma}, V_{s p \sigma}, V_{p p \sigma}$ and $V_{p p \pi}$, and the repulsive potential the form proposed by Ho et al. [18] is adopted.

Our treatment of the effect of the optically created electron-hole plasma is founded on the following physical picture: Due to the action of the laser pulse electrons are excited from occupied to unoccupied levels with a timedependent probability which is proportional to the intensity of the laser field. As a consequence of the extremely fast excitation process, a non-equilibrium distribution of electrons is created. Through electron-electron collisions this electron distribution thermalizes to an equilibrium occupation of the electronic levels. We describe the absorption of energy by the electronic system and its equilibration by:

$$
\begin{aligned}
\frac{d n\left(\epsilon_{m}, t\right)}{d t}= & \int_{-\infty}^{\infty} d \omega g(\omega, t-\Delta t)\left\{\left[n\left(\epsilon_{m}-\hbar \omega, t-\Delta t\right)\right.\right. \\
& \left.\left.+n\left(\epsilon_{m}+\hbar \omega, t-\Delta t\right)-2 n\left(\epsilon_{m}, t-\Delta t\right)\right]\right\} \\
& -\frac{n\left(\epsilon_{m}, t\right)-n^{0}\left(\epsilon_{m}, T_{\mathrm{e}}\right)}{\tau_{1}} .
\end{aligned}
$$

Thus, the electronic distribution is at each time step folded with the pulse intensity function $g(\omega, t)$. This means that at each time step, the occupation of an energy level $\epsilon_{m}$ changes in proportion to the occupation difference with respect to levels at $\epsilon_{m}-\hbar \omega$ and at $\epsilon_{m}+\hbar \omega$. In Eq. (3), constant optical matrix elements are assumed. The second term of Eq. (3) describes the electron-electron collisions that lead to an equilibration of the electronic system with a rate equation of the Boltzmann type for the distribution $n\left(\epsilon_{m}, t\right)$. Hence, with a time constant $\tau_{1}$, the distribution $n\left(\epsilon_{m}, t\right)$ approaches a Fermi-Dirac distribution $n^{0}\left(\epsilon_{m}, T_{\mathrm{e}}\right)$ (with electron temperature $T_{\mathrm{e}}$ ).

This simple approach works very well because for dense electron-hole plasmas extremely short relaxation times $\tau_{1}$ have been found. Chemla and coworkers have reported a carrier thermalization faster than 10 fs in GaAs [19]. As we are not aware of a measured relaxation time in graphite, we use $\tau_{1}=10 \mathrm{fs}$. Note, for such a short thermalization time the exact electronic dynamics leading to electronic equilibrium do not play a significant role for the structural changes we are studying here. The electronic temperature $T_{\mathrm{e}}$ and the chemical potential $\mu$, which appear in the Fermi-Dirac distribution and which are not determined by Eq. (3), need to be fixed by an additional principle. We demand that the nonequilibrium distribution $n\left(\epsilon_{m}, t\right)$ approaches the Fermi-Dirac distribution while conserving the total energy of the system. On the extremely short time scale of $\tau_{1}=10 \mathrm{fs}$, we expect energy loss mechanisms to be negligible.
Using the theory described above we perform simulations on graphite films for a wide range of pulse intensities and durations. This allows us to determine ablation thresholds.

In Fig. 1 we show snapshots of a long trajectory of a graphite film in which melting and subsequent evaporation can be observed. The film consists of $N=576$ atoms. The dynamics shown in this and the following figures are for laser pulses of $\tau=20$ fs duration with a central frequency of $\hbar \omega=3.0 \mathrm{eV}$. We also calculated trajectories at $\hbar \omega=2.0 \mathrm{eV}$ and found the same dynamics. The intensity was determined so that the film absorbs $E_{0}=4.0 \mathrm{eV} /$ atom, corresponding to a carrier density of $n_{c}=6.1 \times 10^{22} \mathrm{~cm}^{-3}$ and a fluence of $F \approx 0.35 \mathrm{~J} / \mathrm{cm}^{2}$ 20. This energy density is high enough to break the bonds of the graphite planes. At $t=80$ fs after the pulse maximum (see Fig. 1 (b)) we see that disorder has developed inside the graphite planes and first bonds are formed between them. At the same time, the first carbon monomer is evaporated from the material, confirming that the graphite planes have been excited above their fragmentation threshold. The following snapshots show the complete disintegration of the graphite planes. In Fig. 1 (c) to (e), a strong volume expansion is observed. In Fig. 1 (c) to (e), four monomers that had already moved far away from the surface were omitted. In the advancing ablation process, further carbon monomers are ejected, and between $\Delta t=520$ fs and $\Delta t=920$ fs we can observe how the expansion of the material takes place especially rapidly in the two surface regions of the film, leading to low densities and the formation of short carbon chains which then start to leave the sample. For the ablation of graphite via the mechanism described above we determined a threshold of $t_{a b 2}=3.3 \pm 0.3 \mathrm{eV} /$ atom, corresponding a fluence of $F_{a b 2} \approx 0.29 \pm 0.03 \mathrm{~J} / \mathrm{cm}^{2}$ [20].

The examination of a graphite film which had absorbed a moderate energy density has produced a surprising result: There is an ablation mechanism which does not lead to a destruction of the graphite planes. The physics of this mechanism is as follows: First, in thermal equilibrium the graphite planes are separated by a very large distance of $d=3.4 \AA$ and consequently their interaction consists only of a very weak van-der-Waals-interaction energy $E \approx 12 \mathrm{meV}$ [21]. Then a photoinduced electronhole plasma causes a very strong vibrational excitation of the graphite layers and at the turning point of their oscillations the atoms of two different layers have a distance of $d \approx 2 \AA$. At this distance the interaction becomes already quite strong and is on the order of $E \approx 1 \mathrm{eV}$. Thus, the laser-induced strong vibrations can lead to collisions of the planes in which momentum in the $z$ direction is transferred. As a result, a surface plane of a graphite sample which usually has zero total momentum can gain enough momentum to leave the surface. 

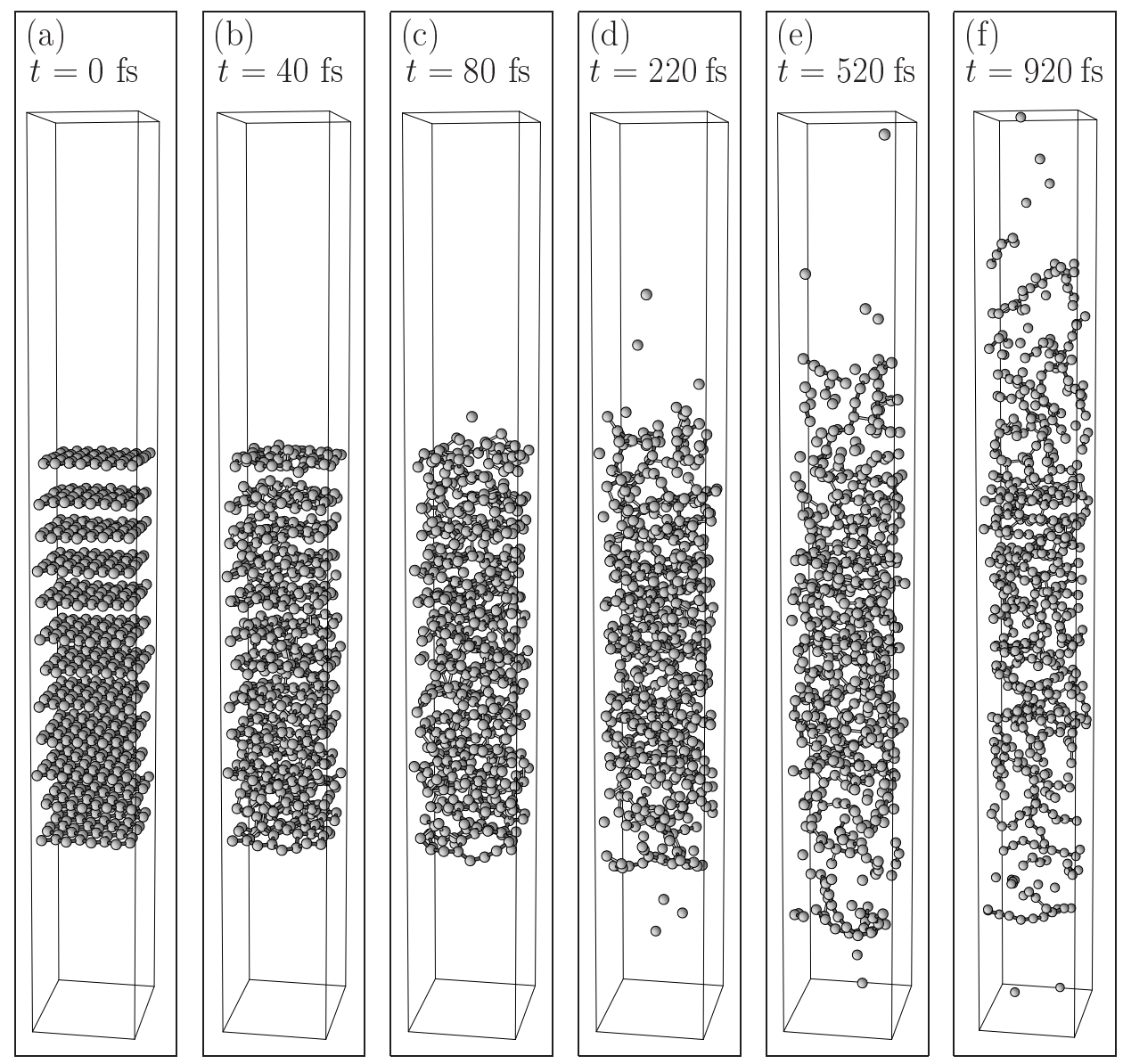

FIG. 1. Ablation of a graphite film for an absorbed energy of $E_{0}=4.0 \mathrm{eV} /$ atom. The laser pulse duration was $\tau=20 \mathrm{fs}$. Note the strong expansion, the formation of a metastable liquid-like state and the emission of carbon atoms and chains.
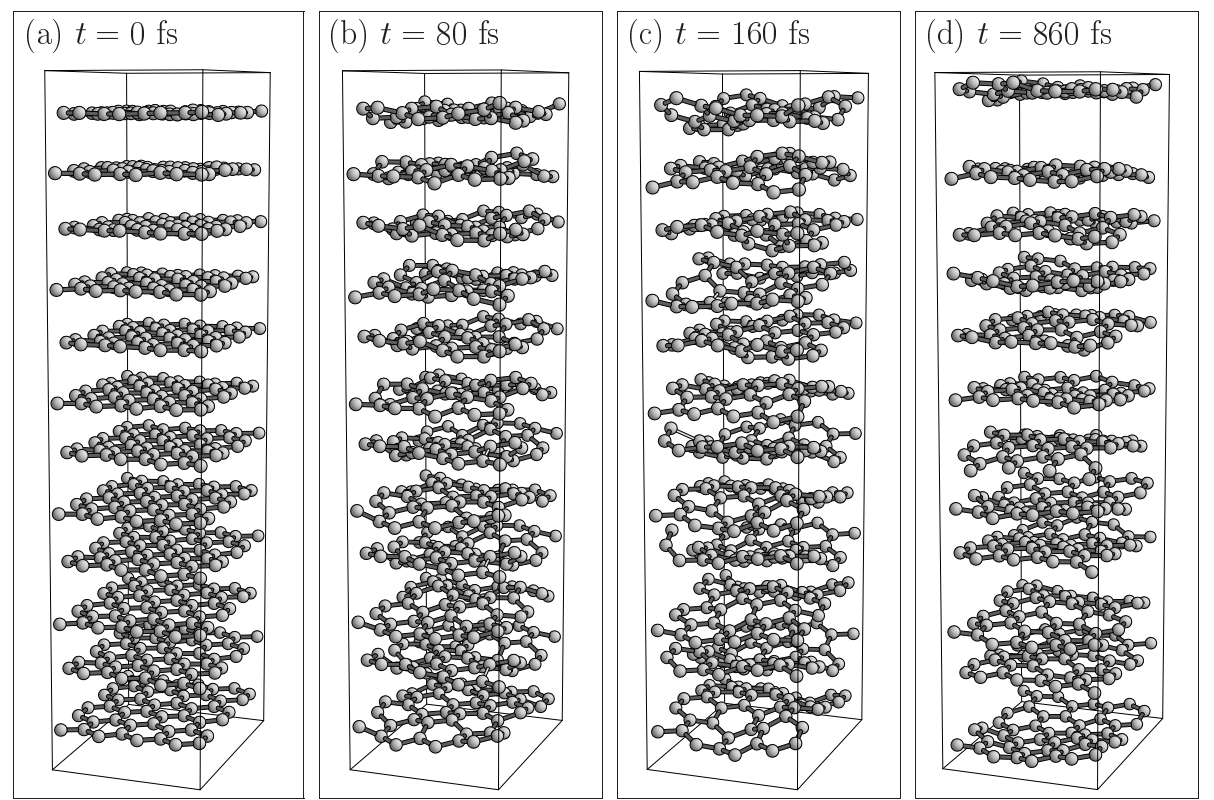

FIG. 2. Ablation of graphite for an absorbed energy of $E_{0}=2.4 \mathrm{eV} /$ atom, below the threshold for the destruction of graphite planes. The pulse duration was $\tau=20 \mathrm{fs}$. Thus, the laser pulse induces strong vibrations of the graphite planes which lead to collisions of the planes. As a consequence, the planes at the top and at the bottom are removed from the surface of the film.

In Fig. 2 we present snapshots of the dynamics of graphite for a $\tau=20$ fs laser pulse with an absorbed energy of $E_{0}=2.4 \mathrm{eV} /$ atom, corresponding to a carrier density of $n_{c}=3.5 \times 10^{22} \mathrm{~cm}^{-3}$ and a fluence of $F \approx 0.21 \mathrm{~J} / \mathrm{cm}^{2}$ 20]. The number of atoms is $N=576$. This trajectory shows the new ablation mechanism. At a deposited energy density that is below the fragmentation threshold of the graphite planes $t_{a b 2}$, we find the ablation of entire graphite planes by the mechanism explained above: The laser pulse leads to a strong vibrational excitation of the graphite layers, with a significant movement of the atoms perpendicular to the graphite planes. This excitation is already strong at $t=80$ fs after the laser pulse maximum, but for the topmost plane it reaches a 
maximum at $t=160 \mathrm{fs}$ (see Fig. 2). At that time the distance between the graphite planes has decreased to approximately $d=1.8 \AA$, and the first and second planes strongly interact. We can speak of a collision of the two topmost graphite planes, which then leads to a momentum transfer. Consequently, the surface plane that had up to that time zero total momentum starts to leave the crystal in positive $z$ direction. In the same way the bottom layer of Fig. 2 can be seen to leave the rest of the film in negative $z$ direction.

This ablation mechanism in graphite has not been described before. It solely relies on the fact that strong vibrational excitation of the graphite planes can increase the interaction between the planes to such a degree that the momentum transferred in this collision process causes ablation of entire planes. It is to be expected, of course, that in reality we are dealing with graphite planes that show slight defects. These would result in large plane fragments being removed from the surface instead of entire graphite planes as the simulation with periodic boundary conditions in the horizontal directions suggests. We estimate the threshold for this ablation mechanism to be $t_{a b}=2.0 \pm 0.4 \mathrm{eV} /$ atom [22], corresponding a fluence of $F_{a b} \approx 0.17 \pm 0.04 \mathrm{~J} / \mathrm{cm}^{2}$ [20].

The absorbed energy $E_{0}=2.4 \mathrm{eV} /$ atom in the example shown in Fig. 2 is high enough to cause the ejection of the first graphite sheet within one picosecond. Note, this absorbed energy is large enough to produce a thermal melting of the removed graphite planes at longer times. This means that although the ablation does not occur via melting, the ablation products might undergo melting after some time.
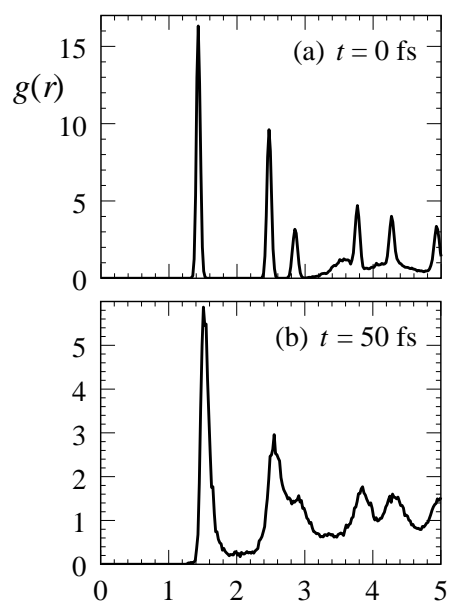
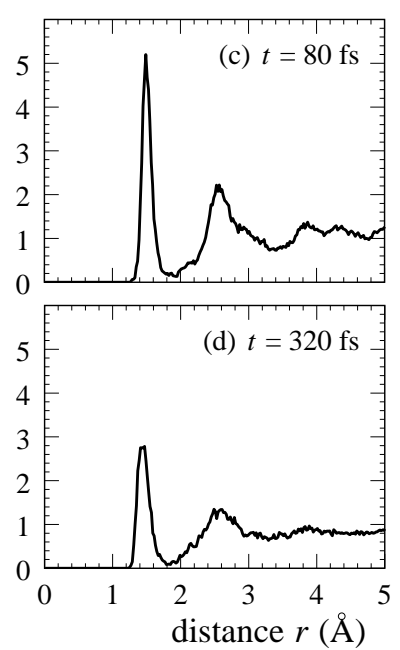

FIG. 3. Pair correlation function $g(r)$ during the melting of a graphite film for an absorbed energy of $E_{0}=4.0 \mathrm{eV} /$ atom. The pulse duration was $\tau=20 \mathrm{fs}$. The pair correlation functions correspond to the same trajectory as Fig. 1. Note, the ordinate scale of (a) is different from that of (b) to (d).
We now analyze in detail the metastable liquid phase induced above $t_{a b 2}$, corresponding to the snapshots of Fig. 1. In Fig. 3 we show the pair correlation functions $g(r)$ during the ultrafast melting of a graphite film. They are calculated as

$$
g(r)=\frac{\langle\Delta N(r)\rangle}{n_{a} 4 \pi r^{2} \Delta r},
$$

where $\Delta N(r)$ is the number of atoms situated at a distance between $r$ and $r+\Delta r$ around a given particle, and $n_{a}=N / \Omega$ is the atomic density of the material. $g(r)$ at the pulse maximum (see Fig. $3(\mathrm{a})$ ) does not yet differ much from $g(r)$ before the pulse. But then a rapid broadening of the structures of $g(r)$ occurs (Fig. 3 (b)) and at $t=80$ fs the pair correlation function of liquid carbon is recognizable (Fig. 3 (c)). Note, because of the expansion of the film this liquid phase has a low density and is characterized by the presence of carbon chains. To characterize this laser induced liquid phase we have determined the order parameter $\Psi=\left(\rho_{4}-\rho_{2}\right) /\left(\rho_{4}+\rho_{2}\right)$ which was proposed by Ree et al. 15] to distinguish between the high density and the low density liquid phase of carbon. Here, $\rho_{2}$ and $\rho_{4}$ correspond to the densities of 2 -fold and 4-fold coordinated carbon atoms determined following Brenner 23]. At a time $t=1 \mathrm{ps}$ after the pulse maximum, we find a value of $\Psi=-0.8$ for the graphite film shown in Fig. 1 confirming that a low density, predominantly 2-fold coordinated liquid carbon phase was produced by the laser excitation.

For a clear check of our predictions pump-probe experiments with a time resolution of less than $100 \mathrm{fs}$ and a structural resolution of a few atomic layers would be required. Such experiments have not been performed on graphite so far. However, the time-resolved reflectivity measurements of laser-irradiated graphite that were reported by Sokolowski-Tinten et al. 24 could be understood qualitatively with the help of our calculations. As in the experiment we can distinguish two different ablation thresholds $\left(t_{a b}, t_{a b 2}\right)$. Our calculated ablation thresholds are in good agreement with the experimental ones $F_{a b}=0.185 \mathrm{~J} / \mathrm{cm}^{2}$ and $F_{a b 2}=0.25 \mathrm{~J} / \mathrm{cm}^{2}$ [24. This demonstrates the validity of our approach using a tight-binding Hamiltonian. It is important to point out that none of the existing $a b$ initio approaches to laser induced melting are able to take the pulse duration and the nonequilibrium electron dynamics into account. Note also that the number of $N=576$ atoms in the MD supercell is not accessible to ab initio calculations [6, 16].

An important result of our approach is that both ablation thresholds are nearly independent of the pulse duration for a range [10fs-500fs]. For fixed pulse duration and increasing energy, qualitative changes only occur at the ablation thresholds. Both the emission of graphite sheets and the ultrafast melting are initiated by strong in- and out-of-plane oscillations of the atoms. 
Summarizing, the microscopic processes leading to laser ablation in graphite films have been studied theoretically. A new ablation mechanism has been observed at energies below the threshold for the destruction of graphite planes. This ablation process is very different from ablation at higher deposited energies, since large graphite plane segments instead of small carbon clusters (chains) are produced. The strong anisotropy of the graphite structure is crucial for this ablation without melting. This process may play a role in the formation of carbon nanotubes by graphite ablation.

This work has been supported by the Deutsche Forschungsgemeinschaft through SFB 450. Our simulations were done on the CRAY T3E at Konrad-ZuseZentrum für Informationstechnik Berlin. We acknowledge helpful discussions with K. Sokolowski-Tinten.

[1] D. H. Reitze et al., Phys. Rev. B 45, 2677 (1992).

[2] P. Stampfli and K. H. Bennemann, Phys. Rev. B 42, 7163 (1990); Phys. Rev. B 49, 7299 (1994).

[3] H. O. Jeschke et al., Phys. Rev. B 60, R3701 (1999).

[4] K. Sokolowski-Tinten et al., Phys. Rev. B 51, 14186 (1995).

[5] P. L. Silvestrelli et al., Phys. Rev. Lett. 77, 3149 (1996).

[6] P. L. Silvestrelli et al., J. Appl. Phys. 83, 2478 (1998).

[7] K. Sokolowski-Tinten et al., Phys. Rev. Lett. 81, 3679
(1998).

[8] K. Sokolowski-Tinten et al., Phys. Rev. Lett. 81, 224 (1998).

[9] J. S. Horwitz et al. (eds.), Laser Ablation, Proceedings of the 5th International Conference, Applied Physics A 69, issue 7 .

[10] B. C. Stuart et al., Phys. Rev. B 53, 1749 (1996).

[11] D. von der Linde et al., J. Opt. Soc. Am. B 13, 216 (1996).

[12] M. Lenzner et al., Phys. Rev. Lett. 80, 4076 (1998).

[13] A. M. Lindenberg et al., Phys. Rev. Lett. 84, 111 (2000).

[14] J. Krüger et al., Laser Physics 9, 30 (1999).

[15] J. N. Glosli et al., Phys. Rev. Lett. 82, 4659 (1999).

[16] A. De Vita et al., Nature 379, 523 (1996).

[17] J. C. Slater et al., Phys. Rev. B 94, 1498 (1954).

[18] C. H. Xu et al., J. Phys. Cond. Mat. 4, 6047 (1992).

[19] W. H. Knox et al., Phys. Rev. Lett. 61, 1290 (1988).

[20] We estimate the fluence from $F=e E_{0} n_{a} d /(1-R-T)$, where $e$ is the Coulomb constant, $n_{a}=1.76 \times 10^{23}$ atoms $/ \mathrm{cm}^{3}$ the atomic density, $d$ the penetration depth, $R=0.17$ the reflectivity and $T=0.65$ the transmission [1]. For $d$ we assume one-photon absorption and apply the Drude formula $d=\lambda / 4 \pi k$ with wave length $\lambda=620 \mathrm{~nm}$ and extinction coefficient $k=1.5$ [1].

[21] J.-C. Charlier et al., Europhys. Lett. 28, 403 (1994).

[22] $t_{a b}$ was estimated taking into account also the van der Waals interaction between the graphite sheets.

[23] D. W. Brenner, Phys. Rev. B 42, 9458 (1990).

[24] K. Sokolowski-Tinten et al., in: Ultrafast Phenomena XI, ed. by T. Elsaesser et al., Springer Series in Chemical Physics 66, 425 (2000).

[25] N. A. Inogamov et al., JETP Lett. 69, 310 (1999).

[26] D. von der Linde et al., Appl. Surf. Science 154, 1 (2000). 